\title{
Experiments on extended performance: Repetition, age, and limited sleep periods
}

\author{
WILSE B. WEBB \\ University of Florida, Gainesville, Florida
}

\begin{abstract}
Four experiments on the effects on performance of extended time periods without sleep and on the effects of limited sleep, that is, naps, within such periods are reviewed. The performance measures included subjective measures and measures of performance on attention/persistence, continuous-production, precision, and cognitive tasks. Repeated periods of sleep deprivation did not result in decreased effects: Older subjects tended to be more vulnerable to sleep-loss decrements, and three different schedules of $4 \mathrm{~h}$ of sleep within a 60 -h sleep-deprivation period had limited differential ameliorative effects.
\end{abstract}

This is a report on a series of experiments on performance during extended time periods without sleep and time periods with limited sleep, or naps. There was an operational orientation in the selection of the variables studied, the measures used, and the analyses conducted.

One experiment assessed the effect of repeated spaced periods of sleep as a potential for reducing performance decrements during sustained operations. Two experiments were concerned with the effect of age on sustained performance. A set of three different schedules of interjected periods of limited sleep, or naps, was used to study their potential as ameliorative resources within extended periods of performance without sleep.

An extensive battery of measures was used to assess a wide range of operationally relevant performances. These included subjective measures and measures of performance on short- and long-term monitoring tasks and continuous-production tasks. A particular effort was made to develop cognitive tasks that would simulate those that might be required of command-level (hence, older) personnel.

Analyses focused on the maximum effects to be anticipated rather than on the measurement of the course-ofperformance changes.

This report includes a description of the methodologies used and summarizes the effects of repeated sleep loss (Experiment 1) and age (Experiments 2 and 3) that have been reported elsewhere. It also presents preliminary analyses (Experiment 4 ) on the effects of performance of three schedules of short periods of sleep (4 h) within extended periods without sleep $(60 \mathrm{~h})$.

\section{METHODOLOGY}

A central overall consideration and several specific ones

The support of U.S. Army Medical Research and Development Command Contract DAMD17-78-8054 and Contract DAMD17-80-0058 are gratefully acknowledged. The author's mailing address is: Department of Psychology, University of Florida, Gainesville, FL 32611. shaped the design, measures, and analyses of these experiments. The central concern was the extent of operational performance decrements associated with sustained operations. The specific issues were: (1) effects on military command-level personnel, who are likely to be older and involved in cognitively demanding tasks, and (2) optimum use of some limited sleep time within continuous operations.

The primary concern, that is, performance during sustained operations, dictated an extensive performance regimen. This was met by programming the presentation and data collection on a video display computer system.

The tasks were administered by a Terak 8510 /a microcomputer system, which incorporates a DEC LSI-11/2 microprocessor. The system controlled each of the two video terminals used to present all visual stimuli and instructions. The programmed system controlled all task sequencing (including scheduling of rest breaks), time- and date-stamped all activities, logged all responses and response latencies, computed mean reaction times, tallied number of correct and incorrect responses, etc.

This regimen also involved extensive repetition of the measures. To meet this problem, our design used a "recovery" baseline, which was a testing period administered after at least 1 week of home sleep recovery. This baseline, then, included all of the practice effects present in the prior deprivation measures. Since our primary orientation was toward operational effects, the deprivation period selected was the time of maximal effects-the final night of deprivation. Although we recognized that this period confounded the "circadian trough" and deprivation, we reasoned that it also reflected the maximum effects of our experimental variable.

The focus on command-level personnel involved older subjects and cognitively demanding tasks. We recruited our subjects by an explanatory letter to an age-graded faculty roster. Our concern with the use of older subjects in sleep deprivation was exhibited by: (1) the requirement 
of a complete physical examination, (2) extending sleep only across 2 nights, (3) permitting sleep immediately after the deprivation period, and (4) beginning with 40 50-year-old subjects before using 50-60-year-old subjects.

To meet the need for cognitively demanding tasks, we performed an extensive (and disheartening) review of the literature. We then obtained the help of a colleague (C. M. Levy), whose specialty area was cognitive psychology combined with high-level skill in computers. We used the initial experiments to modify and extend the battery. A further discussion of these tests and their categorization follows in the Measurement section.

For our interest in optimum time use of limited sleep periods, the basic design included, at appropriate points, nontesting periods that could be utilized for sleep. The decision to use a total of $4 \mathrm{~h}$ of sleep, a decision that predated some of the later work on naps, was arbitrary. The placements were designed to preserve the crucial "nighttime" testing periods and were "commonsensically" set as "preparatory" sleep (sleep prior to the 2nd and 3rd night of sleep deprivation; sleep period $=2200$ $2400 \mathrm{~h}$ ) and "recovery" sleep (sleep following the 1st and 2nd nights of sleep; sleep period $=2000-2200 \mathrm{~h}$ ). There was also a 4-h condition (sleep period $=2000$ $2400 \mathrm{~h}$ ) prior to the 2nd night.

Finally, an obvious but important dictum is emphasized. Deprivation experiments, particularly with older subjects, are best run as subject-operator cooperative enterprises, rather than as adversary ones. The task of the experimenter is to help the subjects maintain wakefulness and performance, rather than to enforce wakefulness. Intrusive sleep events are thereby both subject and experimenter monitored, rather than being the responsibility of the experimenter alone.

\section{Measurements}

The following tests and measures were used. First listed are those that were used in Experiments 1 and 2. Those listed next were used in Experiments 3 and 4. The test sessions and schedules are presented in the description of each experiment.

Stanford Sleepiness Scale. A 7-point scale devised by Hoddes, Zarcone, Smythe, and Dement (1973) was displayed. The subject indicated his sleepiness by referring to an integer, for example, 1 ("almost in reverie; sleep onset soon; lost struggle to remain awake . . or 7 ("feeling active and vital; alert; wide awake"). This scale was given at the beginning of each work session (Scale 1) and again halfway through each session (Scale 2).

Mood Scale. The subjects selected an integer between 1 ("very depressed") and 10 ("elated"). This scale was also presented twice each session (Mood Scales 1 and 2), immediately following each administration of the Stanford Sleepiness Scale.

Auditory Vigilance. This task, devised by Wilkinson (1970), has had repeated use in sleep-deprivation experiments. It requires monitoring 500-msec tones occurring every $2 \mathrm{sec}$ within an $85-\mathrm{dB}$ background noise. In a .5-h test, 20 test tones, each $375 \mathrm{msec}$ in duration, occurred at unsystematic intervals. Hits and false positives were recorded.

Addition. The subject was presented with a column of five three-digit numbers to sum (Wilkinson, 1970). Each set was generated on a random basis subject to the restrictions that each of the five numbers be unique and that no more than two digits be identical within a number. Number of attempts and accuracy were measured during the 30-min self-paced task.

Word Memory. Thirty five-letter words drawn randomly from a pool of 390 five-letter, one syllable, highfrequency words were presented individually for 200 msec, with each presentation followed by a $1,500-\mathrm{msec}$ blank interval. After the last word had been presented, an auditory cue signaled the subject to begin recalling the materials in any order (free recall) as rapidly as possible without regard to typographical accuracy. He was later given an opportunity to edit, that is, to correct the spelling of, his response protocol. One 30-word trial was administered per session. Number of words recalled was scored.

Word Detection. This task was a problem in signal detection. The target was a five-letter low-to-moderatefrequency word and the "noise" background consisted of five-letter nonwords of the same form, that is, consonant-vowel-consonant-vowel-consonant (CVCVC). Fifty of the 100 trials given in a session were noise trials, in which 25 nonwords were presented sequentially at a rate of 10 items/sec. On the remaining signal trials, a target word was unsystematically selected from a pool of 102 items and presented in a randomly determined location between serial positions 12 and 18. The signal trials were interspersed randomly among the noise trials, except that every block of 8 trials contained an equal number of signal and noise trials. The dependent measures were the hit and false-positive rates.

Visual Search. This task was an adaptation of a procedure reported by Neisser (1957). The subject was presented with an array of letters (20 rows, 7 columns) and was to indicate as rapidly as possible when he had detected the presence of a predefined target letter. The subject was instructed to conduct searches from top to bottom and left to right. The target was either " $X$ " or " $Q$ " presented within a background of either rounded letters (e.g., GOCD) or angular letters (e.g., VNKY). An " $X$ " embedded in angular letters or a " $Q$ " surrounded by rounded letters defined a "similar" condition; transposition of these targets defined a "dissimilar" condition. Similar and dissimilar trials were given equally often, in an unsystematic order, during the 80 trials administered per session. The target appeared equally often within each row within each session. The number of correct identifications for similar and dissimilar targets was recorded.

Logical Reasoning. This task (Baddeley, 1968) required the subject to compare a simple sentence (e.g., "A precedes B') and a pictorial relation (e.g., BA) to determine whether the former was an accurate description of 
the latter. The subject was encouraged to respond as quickly as possible. The eight possible combinations of "A" as the subject versus the object of the sentence, the use of "precedes" versus "follows," and affirmative versus negative were combined factorially. When the preceding eight combinations are crossed with the two possible pictorial relations ( $A B$ and $B A), 16$ sentencepicture combinations result, half of which are true. On even-numbered administrations of the task, the right index finger was used to depress a key to indicate a "true" response and the left index finger to indicate a "false" response. On the odd-numbered administrations, this relationship was reversed. This self-paced task lasted $3 \mathrm{~min}$ in each administration. Number of items and number correct were measured.

Remote Associates Test. In this test (Mednick, 1962), the subject was presented with a word triad (e.g., "cookie, sixteen, heart") and asked to generate the word that is associatively related to each (i.e., "sweet"). Twenty-five triads were each displayed for $60 \mathrm{sec}$, after which another set was shown even if the subject had not reported solutions. The number of correct solutions was noted.

Numerical Estimator. This task, described by Irwin, Smith, and Mayfield (1956), was modified to meet the constraints imposed by the laboratory computer. The subject was to imagine two shuffled decks of 500 cards. On each administration of the task, a set of 20 cards was selected and the top pair of cards exposed. Although it was never revealed to the subject, each sample was drawn from a deck that had been generated from a normal distribution with a mean value of 0 and a standard deviation of 2.5. The subject's task was to estimate whether the mean value of the first deck was larger or smaller than the mean for the second deck, and to indicate his confidence in the judgment. The exposed cards were then covered by the next pair of cards, and the subject was allowed to adjust his response after incorporating the new information. This procedure continued without feedback until all pairs from the samples had been exposed. The cards were returned to the master decks and shuffled, and another two sets of 20 cards were withdrawn for the second block of trials. Six blocks of trials were given in each session. Accuracy and confidence were recorded for each judgment.

Object-Uses test. Based upon a task reported by Wilson, Guilford, and Christenson (1953), the procedure required the subject to write all possible ways he could think of to describe the uses of an object. In the present instance, the subject was given a word and an illustrative common usage, and then given $2 \mathrm{~min}$ to generate responses. The subject wrote his responses in script in order to eliminate typing skills as a factor. Six trials were given in each administration of the task, each with a new set of stimulus objects. The number of distinct, plausible responses given to each object was the major dependent measure.

Line Judgment. Adapted from the classic paradigm developed by Crutchfield (1951), the task required the subject to compare a set of three horizontal lines and report which was "neither the longest nor the shortest." The lines were vertically displaced differentially with respect to their origins, and were simultaneously displayed for $1,000 \mathrm{msec}$.

A session began with 15 easy trials in which pilot subjects had been accurate more than $90 \%$ of the time. Subsequently, 45 difficult trials were given in which the difference in line length was small: One line was constructed of 30-45 typewriter underscores, and the other lines differed by \pm 2 underscores. On 15 randomly determined difficult trials, no feedback was given. Feedback of one of two types was given after the remaining trials: On half of these, the subject was informed, "When a group of 400 men your age saw these lines, they reported that Line X was neither the longest nor the shortest," and on the other half, the subject was informed, "The last time you saw these three lines you reported that Line $\mathrm{X}$ was neither the longest nor the shortest." Although these statements were noncontingent upon a subject's response, plausibility was maintained by pointing to the correct response on one-third of the occasions. The task was made demanding intentionally to maximize the opportunity for inducing conforming responses. The dependent variables included number of accurate reports, number of incorrect responses (which were subdivided into conforming and nonconforming responses), judgmental confidence, and response latency.

Unobtrusive Measures. This was a self-initiated task with two components. It was an arbitrarily defined procedural rule: The subject was initially instructed to enter his subject code number (the digit " 1 " or " 2 ") when he began a work session, before he left for a scheduled rest break, and when he returned for each break. The experimental tasks began normally whether or not the subject logged onto the laboratory computer, and the system recorded the subject's action (or failure to act). When a rest break was scheduled, the terminal displayed a message indicating this fact for $30 \mathrm{sec}$. If the subject logged off appropriately within this interval, the system cleared the screen and recorded the subject's action. Otherwise, a failure to respond was noted, the screen was erased, and the system was prepared for the postbreak tasks.

For Experiments 3 and 4, the Numerical-Estimates task and the Unobtrusive Measures were eliminated, and three tasks were added.

Digit Symbol. This test approximated the Digit-Symbol task on the Wechsler-Bellevue Adult Intelligence Scale (Wechsler, 1957). At the top of the computer screen, nine symbols were paired above nine digits. In the middle of the screen, one of the nine symbols appeared. The subject responded by pressing the appropriately matched digit. Correct or incorrect responses prompted a new symbol. The task ran for $10 \mathrm{~min}$ and was self-paced. The number of attempts and the percentage correct were scored.

Long-Term Memory. Bigrams of letters were paired with number pairs. The selection of letters permitted no 
duplicate letters or words, for example, "no"' or "to." The selection of numbers (1-9) also permitted no duplicates. On alternate administrations, the sequence was letter-number of number-letter. Ten pairs were first presented for $1.5 \mathrm{sec}$ each. Then the stem was presented (not in the same order), and the subject attempted to respond with the correct associate. This continued for 10 min or two correct trials. Approximately $1 \mathrm{~h}$ later, the stems were presented twice.

"Learning"' scores were the number of pairs learned in $10 \mathrm{~min}$. The "Recall" measures were percentage of correct pairs recalled on the first trial compared with number of pairs originally learned.

Anagram. Three hundred and thirty common five-letter words were chosen from the Thorndike and Lorge (1944) list and scrambled to form anagrams. Each five-letter set could form only one correct word. The scrambled letters appeared on the screen, and the subject typed in his response. A response, correct or incorrect, resulted in the appearance of a new set of letters. The task continued for $10 \mathrm{~min}$. The number of attempts and percentage correct were scored.

For Experiments 3 and 4 the Visual-Search test was modified to require an identification of the line in which the target appeared.

\section{Subjects}

The younger subjects of these experiments were college students between the ages of 18 and 22 years (Experiments 1, 2, 3, and 4). They were screened with an MMPI, a Cornell Medical Index (CMI), a sleep inven- tory, a personal interview concerned with general personal fitness, and a comprehensive medical examination. These subjects were selected and accepted on a first-come basis if they were within normal MMPI and CMI range, passed the medical examination, and did not maintain atypical sleep habits.

The older subjects were 40-50 years of age (Experiment 2) and 50-60 years of age (Experiment 3). There were 10 subjects in Experiment 2 and 12 in Experiment 3 . They were recruited by letter from the faculty of the University in hopes of approximating military commandlevel personnel. They were also screened by the MMPI, the CMI, the sleep inventory, and a comprehensive medical examination. Two individuals were rejected on the basis of the cardiovascular portion of the physical examination.

\section{EXPERIMENT 1}

\section{The Effects of Repeated \\ Sleep-Deprivation Periods}

This experiment (Webb \& Levy, 1984) assessed the effects of the repetition of spaced periods of sleep deprivation. Six young subjects (18-22 years of age) participated in the schedule of sessions presented in Figure 1. At 3week intervals thereafter, the subjects reported to the laboratory on five occasions. The repeated sessions included a night of laboratory sleep (2300-0700 h) and the sequence outlined in Figure 1 for Days 3, 4, and 5. There was no return testing on Day "12." In short, the subjects remained in the laboratory for testing without sleep

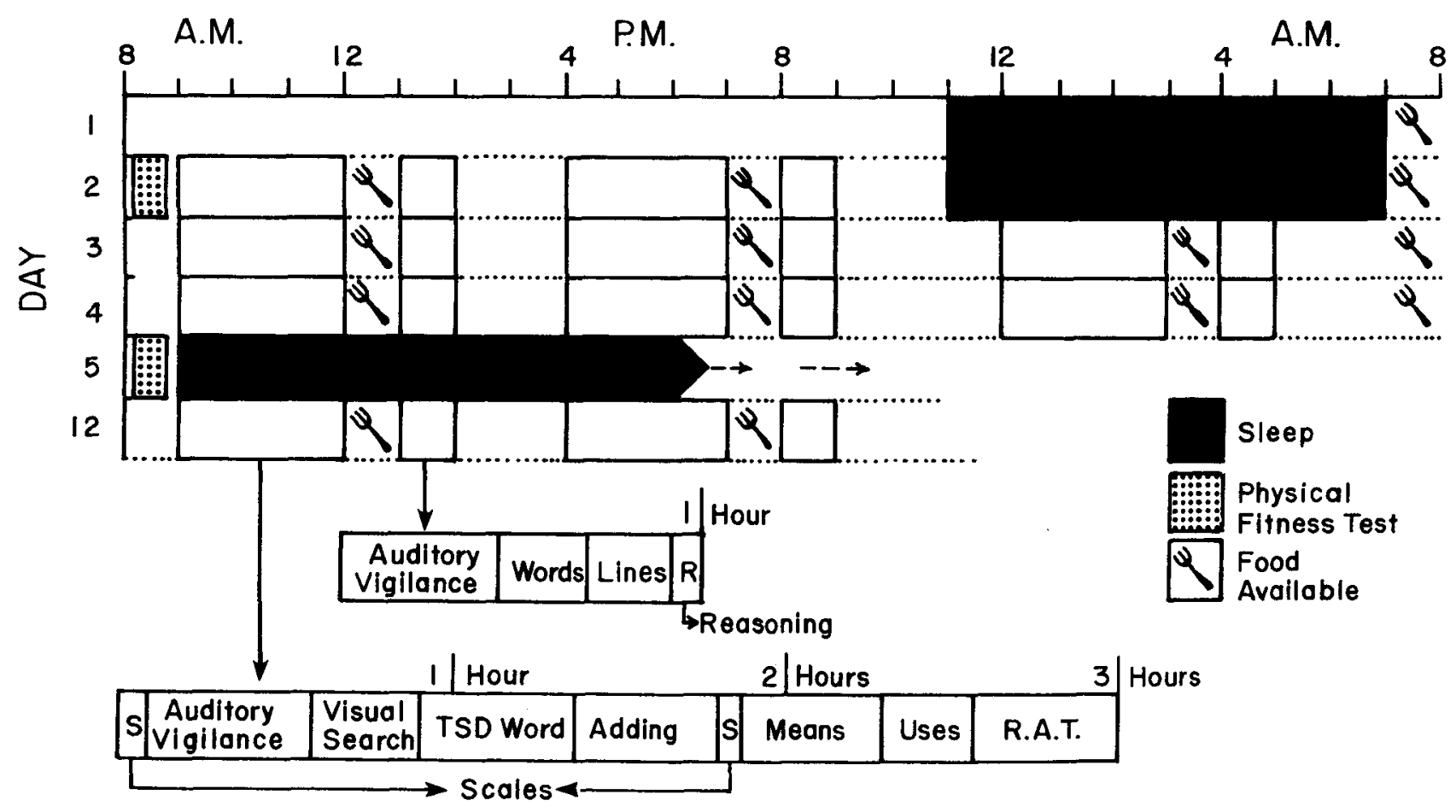

Figure 1. Schedules of experimental protocols. 
for 2 days and 2 nights and slept at the beginning of the 3rd day.

The baseline or nondeprived measures compared with the deprivation measures of Session 1 were those taken after a 12-day recovery period (Figure 1). The baseline measures for the subsequent sessions (Sessions 2, 3, and 4) were from Day 1 measures of each subsequent period; that is, the Day 1 measures for Session 3 served as the nondeprived measures of Session 2, etc. In short, recovery measures after nondeprivation of 12 days (Session 1) and 30 days (Sessions 2, 3, and 4) were used for baseline measures. Thus, the nondeprived comparison scores included all prior learning or additional carryover effects, for example, strategy changes associated with the deprivation measures. The deprivation measures were those from testing periods of maximum deprivation on the 2 nd night without sleep for each session of deprivation. A deprivation $\times$ repetition $\times$ subjects ANOVA was used as a basic analysis procedure.

On those tests that typically are sensitive to sleep deprivation-subjective scales, Auditory Vigilance, and Addition-the subjects showed significant deprivation effects. Line Judgment was not analyzed, and Visual Search was found to be technically flawed. Word Detection and Numerical Estimation did not yield significant results. Deprivation effects were significant for the Word-Usage, Reasoning, and Remote Associates Tasks. However, the cognitive measures, as a group, were less sensitive to deprivation effects.

The subject $x$ deprivation interaction was significant for a number of tests, which, on further analysis, was primarily attributable to the differential responses of individual subjects to deprivation. This finding suggests that some tests may be selected to determine individual sensitivity to sleep-deprivation effects.

In general, either the effects of repetition of sleepdeprivation periods were negligible, or there was a parallel decline in measures. Two alternative hypotheses had been proposed: Repeated deprivation may reduce deprivation effects due to the increased confidence, the development of "coping" mechanisms, and/or the reduction of "stress," or, as had been suggested by Wilkinson (1961) from an earlier experiment, the effects of deprivation would be increased due to lower motivation. In cases in which effects were obtained, the latter position was supported. A number of tests showed a decline from both the baseline and the deprived condition, which suggested decreased motivation across sessions. For several tests (Auditory Vigilance, Word Memory, and Object Usage), there was increasing sensitivity to deprivation relative to baseline as a function of repetition of sleep-deprivation sessions.

Webb and Levy (1984) concluded:

In operational terms, the results indicate that repeated experiences with sleep loss and continuous performance are not likely to result in the development of compensatory or coping tendencies which will affect performance decrements. Rather, such experiences may exacerbate these effects, probably as a result of decrements in motivation. Moreover, we may expect these effects to be task-related in a manner previously noted in single deprivation studies. (pp. 57-58)

\section{EXPERIMENT 2}

\section{Sleep Deprivation, Age, and Performance}

Experiment 2 (Webb \& Levy, 1982) was concerned with the effect of age on performance associated with sleep deprivation. The performance of 6 younger subjects (1822 years) was compared with that of 10 older subjects (40-49 years). The younger subjects were those of Experiment 1 in their first deprivation session.

The schedules and tests were those presented in Figure 1 (Experiment 1). The Day 12 data (performance after 7 days away from the lab) were used for baseline data, and the tests were given after the most extended deprivation during the 2 nd night of deprivation.

Separate subjects $\times$ deprivation analyses were conducted on the younger and older groups, and a Type I ANOVA was used to assess the age $\times$ deprivation effects.

In terms of deprivation effects, it was again found that the subjective scales and the Auditory-Vigilance and Addition tasks yielded robust deprivation effects, and these effects tended to be more reliable for the older age group. Of the cognitively demanding tasks, only Object Uses, Visual Search, and Reasoning yielded significant effects, and these effects were for the older group. Again, the tests sensitive to deprivation were those involving attention and persistence in performance and the subjective measures, whereas the cognitive tests were less sensitive as a group.

Webb and Levy's (1982) conclusions were as follows:

The data empirically indicate that deprivation effects are greater in older subjects both with tests which emphasize speed of performance (e.g., Visual Research, Reasoning, or Object Usage) and those which do not (Auditory Vigilance). However, the simple interpretation of an "age" effect per se is not simply due to differences in initial performance levels in the two groups with the older subjects generally giving higher performance scores. This could reflect higher initial motivation in the older subjects which could not be sustained across the deprivation period. However, this explanation is clouded by the higher subjective measures (Mood and Sleepiness Scales) in the younger subjects. Alternatively, higher performance levels may be more susceptible to sleep deprivation and continuous performance. Unfortunately, we can find no data in the literature on an age controlled group to resolve this possibility. Our subjective observations of the subjects incline us toward a belief in an aging effect. (p. 275)

\section{Additional Findings}

Two further results from the data of Experiment 2 have been published: Webb (1981); Webb, Kaufmann, and Levy (1981).

It was found that a battery of cardiovascular, respiratory, and muscular system measures showed no debilitating effects as a result of 2 nights of sleep deprivation. This study utilized both the 10 40-49-year-old subjects and the 6 younger subjects, which extended the range of physicalfitness measures (Webb, Kaufmann, \& Levy, 1981).

The subjects of Experiment 2 (18-22-year-old and 40- 
49-year-old subjects) were encephalographically recorded during their first period of recovery sleep after 2 nights of sleep deprivation. It was found that although both groups displayed sharply reduced latencies and increased Stage 4 tendencies, the younger group entered slow-wave sleep more quickly (Webb, 1981).

\section{EXPERIMENT 3}

This experiment (Webb, in press) was an extension of Experiment 2 with even older subjects. It compared the performance of $1250-60$-year-old subjects with that of the group of 6 young subjects (18-22 years old). The test battery was modified by the elimination of the NumericalEstimates test and replacing it with the Anagrams test, Digit-Symbol substitution, and Learning/Long-TermMemory task (see Measurements).

The measures were grouped into four categories: subjective, persistence/attention, precision, and cognitive. Again, the data, relative to kinds of measures that are more or less affected by sleep loss, reflect the history of efforts at measuring sleep loss. The subjective and persistence/attention measures were highly sensitive to sleep loss, whereas precision and cognitive processing tasks were less sensitive.

It was noted that there was a general tendency for an increase in variability of scores from the nondeprived to the deprived condition, and greater variability among the older than the younger subjects.

Webb (in press) concluded:

In brief summary the younger subjects showed sharper declines in Subjective Scales. For the Persistence/Attention scores on four tests, the older subjects showed significant declines while the younger subjects did not. One Precision test showed an interaction which resulted from a decline from a higher baseline level in the younger subject to equal performance during deprivation. Two of the Cognitive Demand tasks showed sharper declines by older subjects and two showed the pattern of high baseline performance by younger subjects and sharper declines to equal performance under deprivation. In brief, in these age ranges, where a differential effect was found, the older subjects showed less declines in the subjective ratings but greater declines in tests of Persistence/Attention, Precision, and Cognitive processing. Where there were exceptions, these reflected higher levels of baseline performance by the younger subjects declining to equal performance with the older subjects under deprivation.

\section{EXPERIMENT 4}

In continuous operations, it is possible that opportunities for limited amounts of sleep, that is, "naps," may be available. It is crucial to determine their potential as counterdegradation measures. It appears from the work of Haslam (1982) that limited sleep does offset otherwise total sleep-loss effects in a task-differential manner in field operations. The experiments of Naitoh (1981) indicated that the placement of such short sleep periods may be important.

The present experiment assessed the effects of the placement of $4 \mathrm{~h}$ of sleep within a 72 -h period without sleep.
Three sleep schedules of $4 \mathrm{~h}$ of sleep were used: (1) two sleep periods placed from 0800 to $1000 \mathrm{~h}$ ("recovery" sleep) after the 1st and 2nd night without sleep, (2) two sleep periods of 2-h duration each, placed from 2200 to 2400 h ("preparatory" sleep) prior to the 2 nd and 3rd nights without sleep, and (3) a 4-h evening block from 2000 to $2400 \mathrm{~h}$ before the $2 \mathrm{nd}$ night without sleep. There was a control group that had no sleep. These schedules of sleep, testing, and free time for the groups are diagrammed in Figure 2.

The tests used are described in an earlier section. The arrangements of the tests in batteries and their scheduled administration across the 3 days of testing are shown in Table 1.

There were six young subjects (18-22 years of age) in each of the four groups.

\section{Results}

The deprivation measures of these analyses are the test results taken from the test sessions of maximum deprivation. They were drawn from Batteries A, B, and C, which were administered between 2400 and $0600 \mathrm{~h}$ of the 3rd night of sleep deprivation. The baseline measures were the test results from the recovery-period tests, which were administered between 1000 and $1600 \mathrm{~h}$ after 7 days of home sleep. These non-sleep-deprived measures thus included all of the practice/adaptation effects present for the deprivation measures. For the subjective scales, the summed measures obtained in all three batteries were used. For the Addition test, the summed scores of Batteries $B$ and $C$ were used.

An ANOVA was calculated for the factors of nap type (no nap, morning naps, evening naps, and 4-h naps), deprivation (deprived and nondeprived), and subjects. This analysis yielded effects for nap type ( 3 degrees of freedom), deprivation (1 degree of freedom), nap type $x$ subjects (20 degrees of freedom), deprivation $\times$ nap type ( 3 degrees of freedom), and subjects $\times$ nap type $x$ deprivation (20 degrees of freedom).

Sensitivity to deprivation. Using subjects $\times$ nap type $\times$ deprivation as an error term, the deprivation effect was evaluated for each test. Table 2 lists the measures whose $F$ values for deprivation exceeded the .01 level of confidence and those whose $F$ values did not. Table 2 has been divided into four groupings of tests based on a logical assessment of the measures (Webb, in press). For those tests having significant deprivation effects, the percentage decline of the measures $(100 \cdot B / D$, where $B / D$ = baseline/deprivation) is given.

Test Sensitivity. In accord with our earlier experiments, the subjective measures and the persistence/attention measures continued to reveal robust deprivation effects. Again, however, our extended measures of precision and cognition tests had limited decrements. The previously effective Object-Usage, Visual-Search (similar), and reasoning tests (Experiments 2 and 3 ) were not sensitive. It should be noted that the obtained effects were confined to the older subjects in Experiment 2, and the present data on younger subjects reaffirms the resistance 


\section{PRACTICE \\ TESTING \\ SLEEP}

\begin{tabular}{|c|c|c|c|c|}
\hline $\begin{array}{l}\text { FREE } \\
\text { TIME }\end{array}$ & TESTING & $\begin{array}{l}\text { FREE } \\
\text { TIME }\end{array}$ & TESTING & $\begin{array}{l}\text { FREE } \\
\text { TIME }\end{array}$ \\
\hline
\end{tabular}

\begin{tabular}{|l|l|l|l|l|l|}
\hline I & TESTING & \multirow{2}{*}{ III } & II & \multirow{2}{*}{ TESTING } & $\begin{array}{l}\text { FREE } \\
\end{array}$ \\
& & & III & \\
\hline
\end{tabular}

\begin{tabular}{|c|c|c|c|c|c|}
\hline I & TESTING & $\begin{array}{c}\text { FREE } \\
\text { TIME }\end{array}$ & II & TESTING & $\begin{array}{l}\text { FREE } \\
\text { TIME }\end{array}$ \\
\hline
\end{tabular}

\section{SLEEP OUT $\longrightarrow$}

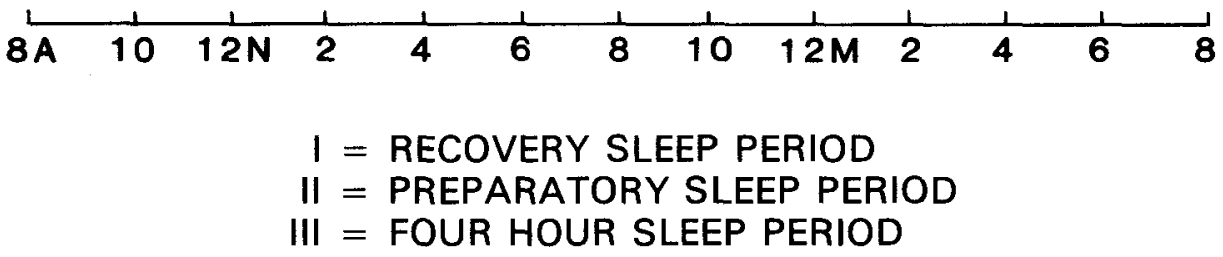

Figure 2. Schedules of sleep periods.

Table 1

Test Schedules for Experiment 4

Test Batteries

Battery A: Sleepiness and Mood Scales ( $3 \mathrm{~min}$ ), Auditory Vigilance (30 min), Word Memory (10 min), Visual Search (20 min), Line Judgment

(12 min), Reaction Time (10 min), Anagrams (10 min), Reasoning (30 min), Scales (3 min)

Battery B: Scales (3 min), Long-Term Memory-Learning (10 min), Addition (30 min), Uses (20 min), Digit Symbol (15 min), Long-Term-Memory Testing (5 min), Scales ( $3 \mathrm{~min})$

Battery C: Scales (3 min), Addition (30 min), Word Detection (30 min), Anagrams (10 min), Scales (3 min)

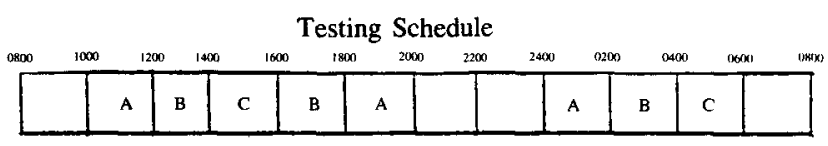

of cognitively demanding tasks to deprivation effects. Of the three cognitive tasks that were added to the battery (Long-Term Memory, Anagrams, and Digit Symbol) only Digit Symbol yielded promising results.

Differential effects. A number of strategies may be used to evaluate the differential effects which involve baseline measures and a measure of an intervening effect
(Cronbach \& Furby, 1970; Harris, 1963). The simplest model recommended by Cronbach and Furby may be applied if the baseline levels of performance on the measures may be considered to be equal. In this model, one tests for differences between the outcome or dependent measures to determine whether the intervening treatments had a differential effect. 
In applying this model to those tests that displayed significant deprivation effects (Table 2), simple treatment ANOVAs were carried out on baseline scores of the four groups to test for the assumption of homogeneity of initial performance level. The null hypothesis could be rejected at the $10 \%$ level of confidence for two of the tests: Vigilance (percentage hits) and Line Judgment (correct feedback).

Next, the treatment ANOVA was applied to the significant-deprivation scores. The null hypothesis could be rejected at the $10 \%$ level of confidence for measures: Stanford Sleepiness Scale 2 (.02), Word-Search hits (.06), Word-Search false alarms (.09), and Line-Judgments (correct).

Table 3 presents the deprivation measures of the Stanford Sleepiness Scale 2, Word-Search hits and false alarms, and the Line-Judgment and Vigilance measures for both the baseline and deprivation measures. These differences were further explored by $t$ tests, and these results are noted in Table 3.
Two significant differences in baseline performance in the Line-Judgment (correct) task and the Vigilance (percentage) task make the interpretation of these data equivocal in this model, but the pattern of the scores indicates that the differences in deprivation scores reflect simply the baseline differences. The remaining tasks (Stanford Sleepiness Scales, Word-Search hits and false-alarms) yield mixed results. The Stanford Sleepiness Scale results can be attributed to the superior performance of the evening-nap group, the Word-Search hits to the superior performance of the morning-nap group, and the WordSearch false alarms to the inferior performance of the nonap group.

A final strategy was used to assess whether scheduled sleep regardless of type had an amelioration effect. The three nap conditions were grouped, and t tests compared these deprivation scores with those of the group receiving no sleep. Three tests that showed more deprivation sensitivity were significant at the .10 level of confidence, indicating significant effects of naps: Addition attempts

Table 2

Test With Significant and Nonsignificant Deprivation Effects $(p<.01)$

\begin{tabular}{llcl}
\hline & \multicolumn{1}{c}{ Significant } & Percent Decline & Nonsignificant \\
\hline Subjective & Sleepiness Scale I & $34 \%$ & \\
& Sleepiness Scale II & $44 \%$ & \\
& Mood Scale I & $26 \%$ & \\
Persistence/Attention & $30 \%$ & Visual Search (S) \\
& Mood Scale II & $20 \%$ & \\
& Addition (N) & $20 \%$ & \\
& Vigilance & $7 \%$ & \\
Wrecision & Word Search & $7 \%$ & Reasoning \%, Digit Symbol \%, Anagrams \%, Vigilance (False) \\
& Visual Search (D) & $3 \%$ & \\
Cognitive & Addition \% & $34 \%$ & Anagrams (N), Reasoning (N), Uses, Line Judgment (Incorrect) \\
& Word Search (False Alarms) & $22 \%$ & Long-Term Memory (Learning), Long-Term Memory (Recall) \\
\hline
\end{tabular}

Table 3

Means and Significance Levels of $t$ Tests of Significant ANOVAs

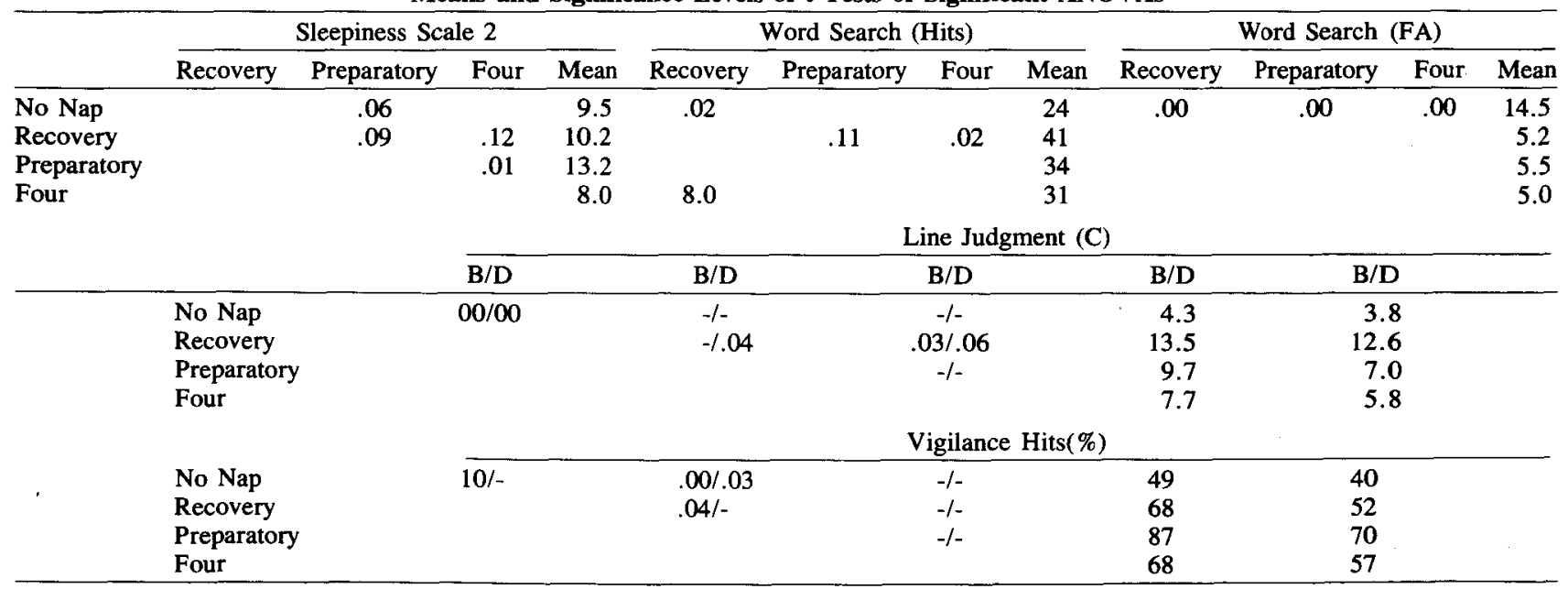

Note $-B / D=$ baseline/deprivation. 
(.04), 101 versus 167 ; Vigilance percentage hits (.08), $40 \%$ versus $60 \%$; Word-Detection (false alarms) (.09), 14.5 versus 5.2 .

\section{DISCUSSION}

Naitoh, Englund, and Ryman (1982, 1983) have reviewed the limited literature on "naps" and performance with particular reference to the use of limited sleep periods in continuous operations as ameliorative resources. Their review points to six variables that may determine performance: (1) length of the deprivation period, (2) length of the nap period, (3) circadian placement of the nap period, (4) time of performance in relation to the nap period (sleep lag), (5) circadian time of task performance, and (6) the performance task.

Within this framework, the present analyses refer to (1) performance during the 3rd night of sleep loss, (2) schedules of two 2-h sleep periods and a 4-h sleep period, (3) evening and morning placement of sleep, (4) performance at least $3 \mathrm{~h}$ after the sleep interval (no sleep lag), (5) during a circadian low period (0000$0600 \mathrm{~h}$ ) and (6) a wide range of tasks.

Only 4 of 13 deprivation-sensitive measures yielded between-treatment differences. For one of these, the difference could be interpreted as reflecting baseline-level differences. There was no consistency among the other three tests: One favored the evening nap, one the morning nap, one the 4-h nap, and one the three-nap conditions. Two additional tests showed a nondifferential effect of sleep versus no-sleep conditions. In general, then, our findings indicated that time placement of two 2-h sleep periods or $4 \mathrm{~h}$ of sleep had few counterdegradation effects. This was based on a wide variety of deprivationsensitive measures (subjective, persistence/attention, precision, and cognitive) taken after substantial periods of wakefulness $(60+h)$ and at a low circadian period $(0000-$ $0600 \mathrm{~h}$ ).

The limited effects of the 2-h naps are in accord with the conclusions of Naitoh et al.'s (1982) review:

In summary, it seems that two hours of recovery sleep, taken at the periods 2000-0200 (Morgan [, Coates, Brown, \& Alluisi], 1973), 2300-1000 (Akerstadt \& Gillberg, 1979), $2300-0130$ (Rosa [, Bonnet, \& Warm], 1983), or 0400-0600 (Naitoh, 1981), has too little restorative power over sleepiness and fatigue resulting from $\mathrm{CW}$ (continuous work).

The results obtained from our 4-h nap condition, however, do not agree with the conclusions of the Naitoh et al. (1982) review: "It could also be concluded ... that a 4hour long recovery period would have considerable restorative power." Two studies (Akerstadt \& Gillberg, 1979; Rosa et al., 1983) that used an awakening-fromsleep design and one (Morgan et al., 1973) that used an immediate-testing design found a significant recovery effect following $4 \mathrm{~h}$ of sleep. In a field study, after 3 days of sleep loss, Haslam (1982) found recovery effects resulting from $4 \mathrm{~h}$ of sleep per night, and Naitoh et al. (1982) reported significant recovery effects on one test (but not on others) from 3-h naps.

Primary differences in our design from those of the others were the interjection of the 4-h nap well before $(24 \mathrm{~h})$ the period of testing, and the test period was conducted during a circadian low period $(0000-0600 \mathrm{~h})$ which extended the period of deprivation. As a consequence, although the 4-h nap may have been "restorative," it was not sufficiently restorative to offset the continued deprivation and circadian effects. It should further be noted that the most comparable studies (Haslam, 1982; Naitoh, 1981) did, in fact, report only limited recovery effects from a single period of 3 or $4 \mathrm{~h}$ of sleep (as used here) and that Haslam found limited recovery during a low circadian period.

In particular, it should be emphasized that these findings of limited nap effects involve a test of terminal effects at the end of 3 nights of deprivation. Preliminary analyses of performance proximal to the nap period indicate clear and substantial recovery effects in the testing period that was proximal to the nap period. In short, an additional factor must be added to the six factors cited by Naitoh (1981): the cumulative deprivation relative to the interjected nap.

From an operational perspective, three points should be noted. First, when buffered from sleep-lag effects, no decremental effects were associated with the interjected sleep period. Second, several tests showed a nondifferential ameliorative effect of naps. Third, within the 3rd night of extended performance, the primary degradation of measures were confined to subjective measures and measures involving relatively long-term attention and persistence of production. Cognitive and short-term attentional tasks showed limited deterioration.

\section{SUMMARY and COMMENTS}

The purpose of this series of laboratory experiments was to assess the potential operational effects of replicated periods of extended operations, age, and limited sleep opportunities on extended performance. A particular effort was made to include measures of performance in the cognitive domain.

The analyses used a "maximum-effect" design by comparing the last deprivation periods that were also in the circadian trough $(0000-0600 \mathrm{~h})$ with a recovery period that followed the extended testing.

The data indicate that repetition of extended performance does not minimize deprivation effects. When effects were found, there was increased sensitivity to deprivation. Older subjects in the age range of 40-60 years of age tended to be more vulnerable to deprivation effects than were younger subjects. However, the differences were not substantial. The presence of $4 \mathrm{~h}$ of sleep in three different schedules revealed only limited differential or ameliorative effects against performance decrements.

Even with the use of a maximal-effect design, although 
the persistence/attentional and subjective tests showed their typical sensitivity to deprivation, the precision and cognitively demanding measures continued to show remarkable resistance to such effects in younger subjects. The most promising tests in this domain were the ShortTerm Memory test and the Digit-Substitution test. These observations must be qualified by noting that the terminal testing period had been preceded by multiple test administrations and that a number of these tests were sensitive only for the older subjects or after repeated deprivation.

Two additional comments about deprivation effects: When the variance of deprivation and nondeprivation scores is evaluated, there is a general tendency for there to be a greater variance associated with the sleep-deprivation scores. In addition, with respect to the process of sleep-deprivation effects, this observation indicates caution. First, analyses of deprivation effects should not be confined to central-tendencies measures. Second, measures that pool variances under the assumption of equality need to be used with caution in refined analyses. A related finding should be noted. In repeated-measures designs, the ANOVAs may yield deprivation $\times$ subjects interactions. These are frequently significant and indicate differential individual responsivity to deprivation. These differences deserve exploration as selection measures and for the further understanding of the sleep-deprivation process.

Finally, I would point to an area of research that, as far as I can determine from the literature, has not been assessed. In research that has explored the differences between older and younger subjects, the baseline measures frequently have yielded a significant difference. In such instances, with appropriate assumptions, these differences may be statistically controlled by the use of analyses of covariance. However, what has not been addressed is a salient question: What is the relationship between level of initial performance and sleep deprivation? I leave this as a challenging research issue.

\section{REFERENCES}

AKERSTADT, T., \& GILLBERG, M. (1979). Effects of sleep deprivation on memory and sleep latencies in connection with repeated awakenings from sleep. Psychophysiology, 16, 49-52.

BADDELEY, A. D. (1968). A 3-min reasoning test based on grammatical transformation. Psychonomic Science, 10, 341-342.

Cronbach, L. J., \& Furby, L. (1970). How we should measure "change"- -or should we? Psychological Bulletin, 74, 68-80.
CRUTChFieldD, R. (1951). Assessment of persons through a quasi groupinteraction technique. Journal of Abnormal and Social Psychology, 46, 577-588.

Harris, E. W. (Ed.). (1963). Problems in measuring change. Madison: University of Wisconson Press.

HASLAM, D. R. (1982). Sleep loss, recovery sleep, and military performance. Ergonomics, 2, 163-178.

Hoddes, E., Zarcone, V., Smythe, H. R., \& Dement, W. C. (1973). Quantification of sleepiness: A new approach. Psychophysiology, 10, $431-436$

IRWIN, F. W., SMITh, W. A. S., \& MAYFIELD, J. F. (1956). Test of two theories of decision in an "expanded judgment" situation. Journal of Experimental Psychology, 51, 261-268.

MEDNick, S. (1962). Associative norms for the creative process. Psychological Review, 69, 220-232.

Morgan, B. B., Coates, G. D., Brown, B. R., Alluisi, E. A. (1973). Effects of continuous work and sleep loss on the recovery of sustained performance (U.S. Army Tech. Memo No. 14-73). Aberdeen Proving Grounds, MD: Human Engineering Laboratory.

NaITOH, P. (1981). Circadian cycles and restorative power of naps. In L. C. Johnson, D. I. Tepas, W. P. Colquhoun, \& M. J. Colligan (Eds.), Biological rhythms, sleep and shift work. New York: Spectrum.

Naitoh, P., Englund, C., \& Ryman, D. (1982). Restorative power of naps in designing continuous work schedules (Tech. Rep. No. 8225). San Diego: Naval Health Research Center.

Naitoh, P., Englund, C., \& Ryman, D. (1983). Extending human effectiveness during sustained operations through sleep management (Tech. Rep. No. 83-13). San Diego: Naval Health Research Center.

NeIsser, U. (1957). Cognitive psychology. New York: AppletonCentury-Crofts.

Rosa, R. R., BonNeT, M. H., \& WARM, J. W. (1983). Recovery of performance during sleep following sleep deprivation. Psychophysiology, 20, 152-159.

THORNDIKE, E. L., \& LORGE, I. (1944). The teacher's word book of 30,000 words. New York: Teacher's College Press, Columbia University.

WEBB, W. B. (1981). Sleep stage responses of older and younger subjects after sleep deprivation. Electroencephalography and Clinical Neurophysiology, 52, 368-371.

WEBB, W. B. (in press). A further analysis of age and sleep deprivation effects. Psychophysiology.

Webi, W. B., KaufmanN, D. A., \& Levy, C. M. (1981). Sleep deprivation and physical fitness in young and older subjects. Journal of Sports Medicine, 21, 198-202.

Webb, W. B., \& Levy, C. M. (1982). Age, sleep deprivation, and performance. Psychophysiology, 19, 272-276.

WEBB, W. B., \& LEVY, C. M. (1984). Effects of spaced and repeated total sleep deprivation. Ergonomics, 1, 45-58.

WECHSLER, D. (1957). Wechsler Adult Intelligence Scale. New York: The Psychological Corporation.

WILKINSON, R. T. (1961). Interaction of lack of sleep with knowledge of results, repeated testing and individual differences. Journal of Experimental Psychology, 62, 263-271.

WILKINSON, R. T. (1970). Methods for research on sleep deprivation and sleep function. In E. Hartmann (Ed.), Sleep and dreaming (pp. 369-380). Boston: Little, Brown.

Wilson, R. C., Guilford, J. P., \& Christenson, P. R. (1953). The measurement of individual differences in originality. Psychological Bulletin, 50, 362-370. 